\title{
Unusual Presentation of Treated Colon Cancer with Extramural Venous Invasion: A Case Report
}

\author{
Ainy Javaid, Afaque Ali, Kashif Siddique, Iqra Zainab \\ Department of Radiology, Shaukat Khanum Memorial Cancer Hospital and Research Centre, Lahore, \\ Pakistan
}

Received: 07 December 2021/Accepted: 20 December 2021

\section{Open access Correspondence:} Afaque Ali, 7A Block R-3, Phase 2, M.A. Johar Town, Lahore 54782, Punjab, Pakistan.

E-mail: afaque.shaikh@hotmail. com

Citation: Javaid A, Ali A, Siddique K, Zainab I. Unusual Presentation of Treated Colon Cancer with Extramural Venous Invasion: A Case Report. J Cancer Allied Spec [Internet]. 2021 Dec 20;8(1):1-5.

https://doi.org/10.37029/jcas. v8i1.451

Copyright: (c) 2022 Javaid, et al.This is an open access article distributed under the terms of the Creative Commons Attribution License, which permits unrestricted use, distribution, and reproduction in any medium, provided the original author and source are credited.

Funding: The authors received no financial support for the research, authorship and/or publication of this article.

Competing interest: $\mathrm{Nil}$.

\begin{abstract}
Introduction: Colon cancer is one of the leading malignancies globally and continues to be one of the most typical causes of cancer-related mortality. The clinical outcome of the disease depends on the primary tumour stage, regional nodal involvement and distant disease dissemination. It often presents with haematogenous spread to the liver at the time of diagnosis. Another factor for increased mortality is the presence of extramural venous invasion. This is exceedingly important as it has significant prognostic significance and helps predict survival. Case Description: A middle-aged female with a recent history of caesarean delivery presented with abdominal pain and occasional constipation, which led to a series of investigations. Initial computed tomography scan showed proximal to mid-transverse colonic tumoural thickening with locoregional lymphadenopathy and solitary distant metastasis in the left hepatic lobe. This was followed by extended right hemicolectomy and hepatic metastasectomy. The patient remained on follow-up and later presented with thrombus formation in the splenoportal circulation. Initially, this was considered a bland thrombus, and the patient was advised a close follow-up. However, the patient was lost to follow and later presented with extensive thrombosis of the portal and splenic veins. Practical Implications: Confident differentiation of the bland versus malignant thrombosis is crucial to ascertain disease stage and appropriate management. Invasive tissue sampling gives a confident diagnosis of benign versus malignant thrombus. However, using a noninvasive imaging modality, we can still distinguish between the two with reasonable certainty.
\end{abstract}

Key words: Bland versus malignant thrombus, cancer-related mortality, colon cancer, extramural venous invasion, haematogenous spread, thrombosis

\section{Introduction}

Colorectal cancer accounts for the third most frequent cancer and the fourth most common cause of cancer-related mortality. ${ }^{[1]}$ The treatment options and survival benefits depend on the tumour stage at the time of presentation, which depends on the extent of colonic wall involvement, locoregional nodal disease and presence of distant metastasis according to the tumour, node and metastasis Dukes classification. ${ }^{[1,2]}$ Approximately $20 \%$ of the patients present with metastatic disease at the 
time of presentation. ${ }^{[2]}$ In patients with colorectal cancer, the liver is the most involved site of distant metastasis. ${ }^{[2]}$ In addition, the dissemination of tumour cells outside the bowel wall and into the venous system is an important aspect that has significant impacts in defining the clinical outcomes and discerning the overall prognosis. ${ }^{[1,2]}$ Venous invasion is a relatively less frequent occurrence in colorectal cancer, accounting for $30 \%$ of cases of rectal cancer. However, venous infiltration in the case of colon cancer is an even more rare occurrence. ${ }^{[3]}$

To date, radical surgical resection in colon cancer remains a conventional treatment. However, early treatment with neoadjuvant chemoradiotherapy is effective for locally advanced cases. ${ }^{[4]}$ Colorectal liver metastases are usually accompanied by microscopic tumour invasion into the intrahepatic portal vein. However, the incidence of macroscopic tumour thrombus in the trunk of the portal vein is rare. ${ }^{[5,6]}$ Bland thrombus is often encountered within the portal venous system. It can occur in the presence and absence of a tumour thrombus. In instances, they may coexist and make the differentiation between the two a crucial entity. ${ }^{[6]}$

This case report presents a rare case of colon cancer in a 37-year-old female, on surveillance, presenting with extramural venous invasion (EMVI). The clinical presentation, radiographic findings, histological diagnosis and management are discussed in the light of the literature review. The hospital Institutional Review Board granted a waiver of informed consent to publish this case report.

\section{Case Description}

A 37-year-old female presented in the outpatient clinic with the complaints of abdominal pain and occasional constipation after 6 weeks of her caesarean section. The episodes of constipation became frequent 4 weeks before her visit. There was an additional history of weight loss of $7 \mathrm{~kg}$ during her postpartum period, which she attributed to her expected weight loss post-delivery. The patient had no history of abdominal or pulmonary tuberculosis, hypertension, diabetes or other systemic medical conditions. The patient belonged to a lower-middle class socioeconomic status and did not have any family history for cancer in blood relatives.

\section{Diagnosis and management}

Baseline biochemical tests and investigations were performed, including digital rectal examination, proctoscopy and colonoscopy, followed by imaging including computed tomography (CT) scans. Clinical examination depicted visible pallor and tenderness in the epigastric region. Digital rectal examination and proctoscopy were unremarkable and did not reveal any abnormal rectal growth. Colonoscopy examination was performed a week later, which showed circumferential growth involving the transverse colon and hepatic flexure spanning a length of approximately $8 \mathrm{~cm}$. Tissue samples were obtained in the same sitting. Histological studies of the biopsied tissues were significant for moderately differentiated adenocarcinoma involving the hepatic flexure and proximal two-thirds of the transverse colon. The ascending colon showed no dysplasia or invasive tumour.

A staging CT scan of the chest, abdomen and pelvis was done, which showed a long segment circumferential mass-like thickening of the proximal to the middle transverse colon with associated pericolonic lymp nodes and $6 \mathrm{~mm}$ metastatic nodule in the segment IV B, making it T3, N2, M1 disease (figure 1). The case was thoroughly discussed in a multidisciplinary conference. The consensus was to proceed with extended right hemicolectomy and hepatic metastasectomy.

Subsequently, laparoscopic-assisted extended right hemicolectomy and hepatic metastasectomy were performed. Tissue sampling from the peritoneal nodule and falciform ligament was also obtained. Post-operative specimen histology was compatible with the pre-operative diagnosis of moderately differentiated adenocarcinoma involving an $85 \mathrm{~mm}$ long colonic segment, invading 
through the muscularis propria into the pericolonic fat pT3. Proximal and distal resection margins were free of tumour. Two out of 26 lymph nodes were positive for metastasis. Resected hepatic nodule showed metastatic disease with the tumour at a distance of $6 \mathrm{~mm}$ from the cauterised inked margin. The omental biopsy was positive for metastasis.

This was followed by five cycles of adjuvant chemotherapy over 4 months. The patient subsequently developed severe abdominal pain and vomiting with altered mental status, leading to the termination of further chemotherapy. Reevaluation magnetic resonance imaging (MRI) brain with contrast was performed, which showed nodular leptomeningeal enhancement concerning leptomeningeal carcinomatosis. However, the cerebrospinal fluid cytology was negative for disease involvement.

A re-evaluation CT scan was performed, which revealed a long filling defect in the superior mesenteric vein and proximal splenic vein extending proximally into the portal vein confluence with associated surrounding fat stranding (figure 2). This was considered bland thrombosis with associated mesenteric congestion secondary to portal vein occlusion with no definite signs suggesting tumour involvement. Hepatic metastasis involving segments VII and VIII was demonstrated.

She was kept on thrombolytic medication, but her symptoms did not improve. Due to her worsening health status, repeat CT was obtained at a 1.5-month interval to see the disease status, revealing progressive heterogeneously enhancing portal vein thrombosis with significant vascular expansion and extension into the superior and inferior mesenteric veins (figure 3 ). The thrombus was seen to extend proximally to the confluence of the right and left portal vein branches. There was a contiguous extension of the thrombus within the splenic vein. Significant adjacent collateralisation was noted. Associated mass effect was evident on the pancreatic head, uncinate process and body with no intervening fat plane. Furthermore, significant heterogeneity was noted in the periportal region with nodular soft-tissue morphology making a confluent mass-like configuration measuring 2.5 $\times 2.2 \mathrm{~cm}$, insinuating into the aortocaval recess. Serosal deposit along the anterior aspect of the left lobe of the liver and a few enlarged lymph nodes at the porta were among the new findings. Hepatic metastases were reidentified.

The patient was rediscussed in a multidisciplinary tumour board, and a decision was made to put the patient on the palliative pathway. The patient had a follow-up visit at 6 months with persistent symptoms of abdominal discomfort and generalised toniccolonic seizures and was managed conservatively.

\section{Discussion}

Haematogenous and lymphatic spread is responsible for metastasis from colorectal cancer, most commonly involving the liver and lung. ${ }^{[6]}$ Over $50 \%$ of all patients with colorectal cancer develop liver metastasis during their disease course. ${ }^{[7]}$ Microscopic tumour invasion into the intrahepatic portal vein is common. However, macroscopic tumour thrombus in the portal branch is rare. Little is known about the incidence, clinical characteristics or outcome of surgical treatment for patients with such tumour thrombi. ${ }^{[4]}$ The presence of EMVI has prognostic value, indicating distant disease recurrence. ${ }^{[8]}$ It often is an under-reported finding with considerable variability in its reporting.

Further venous invasion is also not separately documented from the umbrella term of lymph vascular invasion. This may not be appropriate as this information can be critical in predicting overall survival benefits. The presence of which is associated with poor outcome. ${ }^{[9]}$

Venous thrombosis can be classified as neoplastic or bland. The characterisation of thrombosis as neoplastic versus bland is crucial for accurately determining tumour stage and treatment options and often poses a diagnostic challenge..$^{[10]}$

Image-guided fine-needle aspiration is a sensitive and safe investigation to distinguish neoplastic 


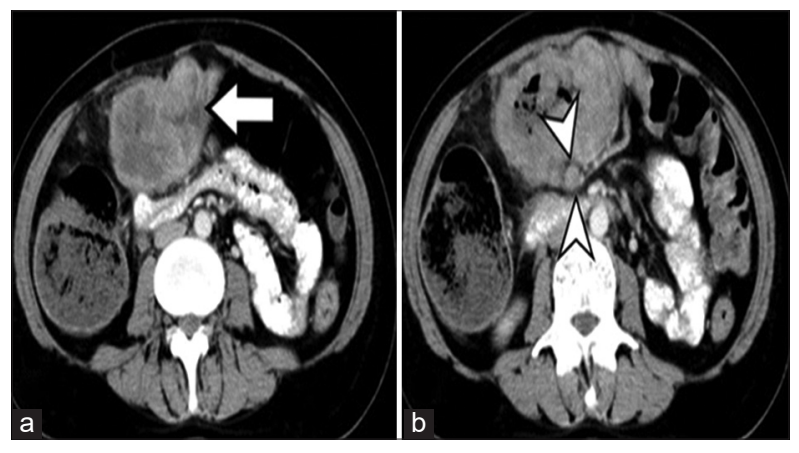

Figure 1: (a) Pre-operative computed tomography scan with axial sections through the abdomen with intravenous contrast showed long segment circumferential masslike thickening of the proximal to the mid-transverse colon (white arrow) (b) with pericolonic fat stranding and associated multiple pericolonic lymph nodes (arrowheads)
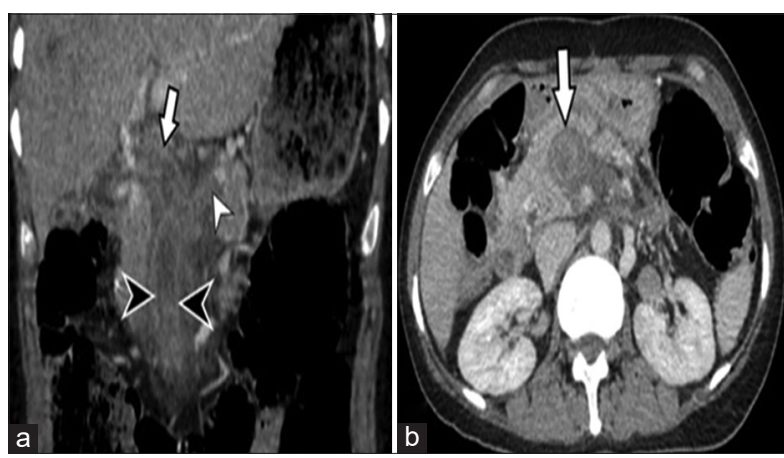

Figure 2: Post-operative computed tomography of abdomen in portovenous phase obtained at 4-month interval. Coronal reformatted (a) and axial images (b) showed long segment filling defect in the superior mesenteric vein (black arrowhead) (a) and splenic vein (white arrowhead) (a) extending into the main portal vein (white arrowhead) (b) with associated surrounding fat arborisation

from the bland thrombus and is considered the reference standard. ${ }^{[11]}$ Although it is considered a safe and sensitive (up to $93 \%$ sensitivity) technique, portal vein biopsy is an invasive procedure associated with complications such as bleeding and the possibility of false-negative results due to sampling error. As a result, there has been increasing reliance on imaging studies such as CT, $\mathrm{MRI}$ and positron emission tomography-CT for the characterisation of venous thrombosis. In particular,

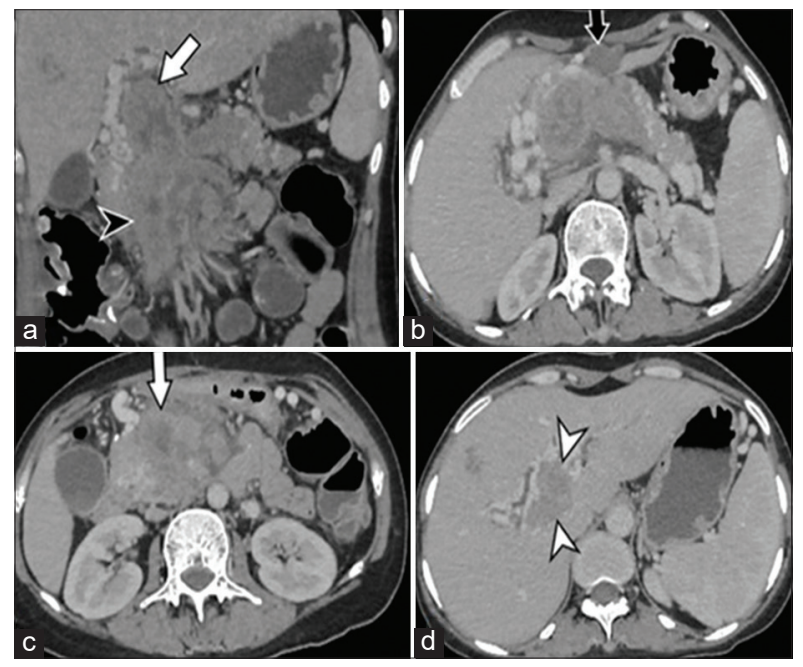

Figure 3: Computed tomography abdomen with coronal reformatted (a) and axial sections ( $b, c$ and $d$ ) in portovenous phase at the level of portal vein showed progressive heterogeneously enhancing superior mesenteric and portal vein thrombosis with significant vascular expansion (white arrow) ( $a$ and $c$ ) and extension into the confluence of the right and left portal vein branches and splenic vein (white arrowhead) (d) and serosal deposit of $2 \mathrm{~cm}$ along the anterior aspect of the left lobe of the liver (black arrowhead) (b)

CT remains a useful non-invasive imaging modality to distinguish bland versus tumour thrombosis with reasonable certainty. ${ }^{[12]} \mathrm{CT}$ texture analysis and density acquisitions allow confident differentiation of neoplastic and bland thrombus. ${ }^{[13]}$

Cardinal features inadequately characterising benign from malignant thrombosis include venous expansion, intrathrombus neovascularity and direct tumoural infiltration. ${ }^{[12]}$ In addition, the presence of enhancement, the most specific sign and diffusion restriction on MRI also provide information regarding the nature of the venous thrombus. ${ }^{[14]}$

Diffusion-weighted imaging has more sensitivity and specificity in distinguishing bland from tumoural thrombosis. One of the studies revealed an apparent diffusion coefficient (ADC) of portal vein thrombi, and the ADC ratio was higher in bland portal vein thrombosis than in any case of neoplastic portal vein thrombosis. ${ }^{[7]}$ 
This case report is one of the few cases of colonic cancer presenting with frank EMVI after surgical resection of the primary malignancy. Timely and accurate detection can help pave the path for alternative treatment options and predict the overall prognosis.

\section{Acknowledgments}

None.

\section{References}

1. Mármol I, Sánchez-de-Diego C, Dieste AP, Cerrada E, Yoldi MJ. Colorectal carcinoma: A general overview and future perspectives in colorectal cancer. Int J Mol Sci 2017;18:197.

2. Hugen N, Van de Velde CJ, De Wilt JH, Nagtegaal ID. Metastatic pattern in colorectal cancer is strongly influenced by histological subtype. Ann Oncol 2014;25:651-7.

3. Dawson H, Kirsch R, Driman DK, Messenger DE, Assarzadegan N, Riddell RH. Optimizing the detection of venous invasion in colorectal cancer: The Ontario, Canada, experience and beyond. Front Oncol 2015;4:354.

4. Kim TH, Woo S, Han S, Suh CH, Vargas HA. The diagnostic performance of MRI for detection of extramural venous invasion in colorectal cancer: A systematic review and meta-analysis of the literature. Am J Roentgenol 2019;213:575-85.

5. Yamamoto N, Sugano N, Morinaga S, Kanazawa A, Inagaki D, Shiozawa $M$, et al. Massive portal vein tumor thrombus from colorectal cancer without any metastatic nodules in the liver parenchyma. Rare Tumors 2011;3:148-9.

6. Choi PW, Kim CN, Chang SH, Chang WI, Kim CY, Choi HM. Cardiac metastasis from colorectal cancer: A case report. World J Gastroenterol 2009;15:2675-8.

7. Lee DH, Lee JM, Hur BY, Joo I, Yi NJ, Suh KS, et al. Colorectal cancer liver metastases: Diagnostic performance and prognostic value of PET/MR imaging. Radiology 2016;280:782-92.

8. Messenger DE, Driman DK, Kirsch R. Developments in the assessment of venous invasion in colorectal cancer: Implications for future practice and patient outcome. Hum Pathol 2012;43:965-73.

9. Gibson KM, Chan C, Chapuis PH, Dent OF, Bokey L. Mural and extramural venous invasion and prognosis in colorectal cancer. Dis Colon Rectum 2014;57:916-26.

10. Sherman CB, Behr S, Dodge JL, Roberts JP, Yao FY, Mehta N. Distinguishing tumor from bland portal vein thrombus in liver transplant candidates with hepatocellular carcinoma: The A-VENA Criteria. Liver Transpl 2019;25920:207-16.

11. Vilana R, Bru C, Bruix J, Castells A, Sole M, Rodes J. Fine-needle aspiration biopsy of portal vein thrombus: Value in detecting malignant thrombosis. AJR Am J Roentgenol 1993;160:1285-7.

12. Tublin ME, Dodd GD $3^{\text {rd }}$, Baron RL. Benign and malignant portal vein thrombosis: Differentiation by CT characteristics. AJR Am J Roentgenol 1997;168:719-23.

13. Canellas R, Mehrkhani F, Patino M, Kambadakone A, Sahani D. Characterization of portal vein thrombosis (neoplastic versus bland) on CT images using software-based texture analysis and thrombus density (Hounsfield units). Am J Roentgenol 2016;207:W81-7.

14. Catalano OA, Choy G, Zhu A, Hahn PF, Sahani DV. Differentiation of malignant thrombus from bland thrombus of the portal vein in patients with hepatocellular carcinoma: Application of diffusionweighted MR imaging. Radiology 2010;254:154-62.

\section{Authors' Contributions}

Conceived and designed the analysis: AJ, AA and KS. Collected the data: AJ and IZ. Contributed data or analysis tools: AA, KS and IZ. Performed the analysis: AA, AJ and IZ. Wrote the paper: AJ, $A A, K S$ and $I Z$. 